\title{
ED and poison center surveillance for the Great American Solar Eclipse in Oregon
}

\author{
Laurel Boyd $^{* 1}$, Sandy Giffin ${ }^{2}$, Meredith Jagger ${ }^{1}$ and Melissa Powell ${ }^{1}$ \\ 'Oregon Public Health Authority, Portland, OR, USA; '2Oregon Poison Center, Portland, OR, USA
}

\section{Objective}

Identify surveillance priorities for emergency department (ED) and Oregon Poison Center (OPC) data ahead of the 2017 Great American Solar Eclipse gatherings in Oregon and create a suite of queries for use in the Health Intelligence Section of the Oregon Public Health Division (OPHD) Incident Management Team (IMT).

\section{Introduction}

Oregon's statewide syndromic surveillance system (Oregon ESSENCE) has been operational since 2012. Non-federal emergency department data (and several of their associated urgent care centers) are the primary source for the system, although other data sources have been added, including de-identified call data from OPC in $2016(1)$.

OPHD epidemiologists have experience monitoring mass gatherings (2) and have a strong relationship with OPC, collaborating on a regular basis for routine and heightened public health surveillance. Nevertheless, surveillance for the Great American Solar Eclipse (August 2017) presented a challenge due to the 107 reported simultaneous statewide eclipse-watching events planned for the day of the eclipse (some with estimated attendance of greater than 30,000 people and most in rural or frontier regions of the state).

Scientific literature is limited on mass gathering surveillance in the developed world (3), particularly in rural settings (4), so OPC and OPHD worked together to develop a list of health conditions of interest, including some that would warrant both an ED visit and a call to OPC (e.g., snake bites). Monitoring visits in both data sources in would allow for assessment of total burden on the healthcare system, especially in the case of snake bites where only specific bites require administration of anti-venom.

\section{Methods}

Ahead of the planned mass gatherings, OPHD Health Intelligence and OPC compiled a list of expected risks from the literature $(4,5)$ and input from members of the IMT including the Public Information Officer, who monitored media for stories about health. Priority health conditions presented a clear risk to public health (e.g., limited supply of snake anti-venom warranted surveillance of snake bites) or were the subject of substantial media coverage. Query development focused on risks that had specific, well-defined health effects and that would be captured by syndromic ED and OPC data.

During an enhanced surveillance period (8/18-8/24), OPHD Health Intelligence reviewed and interpreted trends in common queries with $\mathrm{OPC}$ and disseminated a daily statewide surveillance report.

\section{Results}

OPHD and OPC created four new queries for both ED and OPC data streams: snake bites, psychedelic mushrooms, $2^{\text {nd }}$ and $3^{\text {rd }}$ degree body burns and eye-related calls and visits. ED queries used chief complaint, discharge diagnosis, or triage note. OPC queries used generic code, therapy and clinical effect.

From $8 / 18-8 / 22$, OPHD Health Intelligence distributed daily surveillance reports to the OPHD IMT and external partners. An increased in eye-related injuries was identified on the day after the eclipse, prompting OPHD Health Intelligence to consult with OPC.
ED surveillance data indicated that the increase in eye-related visits was likely a seasonal trend. OPC staff reviewed the charts of patient calls captured by the query and concluded the calls were not related to retinal issues from looking at the sun. No other trends were noted in the joint OPHD/OPC queries.

\section{Conclusions}

OPHD Health Intelligence piloted four new queries for surveillance during this mass gathering event and exercised the process for disseminating trend information from OPC and ED data. The eclipse event was fairly quiet and very few trends of note were captured by surveillance. Prior to this event, OPC data had not been a part of the Health Intelligence surveillance plan. However, assessing trends in OPC data provides an opportunity to better understand trends seen in ED data (e.g., whether or not a surge in ED visits for snake bites is accompanied by a surge in OPC calls for anti-venom is meaningful). By building a process to review disparate data in tandem, OPHD and OPC strengthened regional surveillance for this event. Applicable queries will continue to be used for planned event surveillance and several additional queries are currently under development.

\section{Keywords}

Poison Center; Mass Gathering; Eclipse; Syndromic Surveillance; Query Creation

\section{Acknowledgments}

Jamie Bash, Richard Leman, Emilio Debess, David Lehrfeld, Eric Gebbie, DeWayne Hatcher, Paul Cieslak

\section{References}

1. Laing R, Powell M. Integrating Poison Center Data into Oregon ESSENCE using a Low-Cost Solution. 2017;9(1):2579.

2. Jagger MA, Jaramillo S, Boyd L, Johnson B, Reed KR, Powell M. Mass Gathering Surveillance : New ESSENCE Report and Collaboration Win Gold in OR. 2017;9(1):2579.

3. Steffen R, Bouchama A, Johansson A, Dvorak J, Isla N, Smallwood C, et al. Non-communicable health risks during mass gatherings. Lancet Infect Dis. 2012;12(2):142-9.

4. Polkinghorne BG, Massey PD, Durrheim DN, Byrnes T, MacIntyre CR. Prevention and surveillance of public health risks during extended mass gatherings in rural areas: The experience of the Tamworth Country Music Festival, Australia. Public Health [Internet]. 2013;127(1):32-8. Available from: http://dx.doi.org/10.1016/j.puhe.2012.09.014

5. Lombardo JS, Sniegoski CA, Loschen WA, Westercamp M, Wade M, Dearth S, et al. Public health surveillance for mass gatherings. Johns Hopkins APL Tech Dig (Applied Phys Lab. 2008;27(4):347-55.

\section{*Laurel Boyd}

E-mail: laurelhifi@gmail.com 